\title{
The marshall-olkin ikum distribution
}

\begin{abstract}
In this paper, a new class of continuous model using Marshall-Olkin Extended method is introduced. We describe shapes of probability density function and hazard rate function. Also some sub-models and relation between new model and other distributions are presented. The moments, moment generating function, quantiles and mode are derived. The parameters are estimated by maximum likelihood method.
\end{abstract}

Keywords: hazard rate, kumaraswamy distribution, marshall-olkin family, moments, moment generating function
Volume 7 Issue I - 2018

\author{
Lishamol Tomy,' Jiju Gillariose 2 \\ 'Department of Statistics, Deva Matha College, India \\ ${ }^{2}$ Department of Statistics, St.Thomas College, India
}

\begin{abstract}
Correspondence: Lishamol Tomy, Department of Statistics, Deva Matha College, Kuravilangad, Kerala-686633, India, Email lishatomy@gmail.com
\end{abstract}

Received: November 09, 2017| Published: January II, 2018

\section{Introduction}

Finding alternative to most well known classical family of distributions has become a topic of high importance in academic research due to their striking properties. An increasing interest can be observed for the art of adding parameter to some existing distributions as one parameter family of distributions is not sufficient to handle various real contexts. There is a vast amount of literature on method of introducing new distributions. Several statistical experts have mentioned similar processes (exponentiated family of distributions (by Gupta et al., ${ }^{\text {) }), ~ K u m a r a s w a m y ~ f a m i l y ~ o f ~ d i s t r i b u t i o n s ~(b y ~ C o r d e i r o ~}$ and Castro $^{2}$ ), Kummer beta generalized family of distributions (by Pescim et al., ${ }^{3}$ ), geometric exponential-Poisson family of distributions (by Nadarajah et al., ${ }^{4}$ ), exponentiated T-X family of distributions (by Alzaghal et al.,5), Weibull generalized family of distributins (by Bourguignion et al., ${ }^{6}$ modified beta generalized family of distributions (by Nadarajah et al., ${ }^{7}$ ) and exponentiated exponential-Poisson family of distributions (by Risti $\mathrm{c}^{\prime}$ and Nadarajah). ${ }^{8}$ ) with applications in various factual contexts as inclusion of new parameter to existing family of distributions increases flexibility of the distributions. Also data with a high degree of skewness and kurtosis can be modeled. Again it is helpful in improving the goodness of fit of proposed generalized family of distributions. We may use various methods to add new parameters for expanding family of distributions. Marshall and Olkin ${ }^{9}$ introduced a general method, the resulting distribution is called Marshall-Olkin Extended (MOE) family of distributions, its cumulative density function (cdf) $G(x)$ and probability density function (pdf) $g(x)$ are given by the following formulae,

$$
\begin{array}{r}
G(x, \alpha)=\frac{F(x)}{\alpha+(1-\alpha) F(x)}, x \in R, \alpha>0 \\
g(x, \alpha)=\frac{\alpha f(x)}{(\alpha+(1-\alpha) F(x))^{2}}, x \in R, \alpha>0
\end{array}
$$

where $F(x)$ is cdf and $\alpha$ is a tilt parameter of a random variable $X$. If $F(x)$ has the hazard rate function $r(x)$ then the hazard rate function of MOE family is given by

$$
h(x, \alpha)=\frac{r(x)}{\alpha+(1-\alpha) F(x)}, x \in R, \alpha>0
$$

Study on inverted distributions is another area of interest. There are a lot of research study about inverted distributions such as Calabria and Pulcini ${ }^{10}$ studied the inverse Weibull, AL-Dayian ${ }^{11}$ introduced the inverted Burr Type XII distribution, Abd EL-Kader et al., ${ }^{12}$ described the inverted Pareto type I distribution, AL-Dayian ${ }^{13}$ discussed inverted Pareto Type II distribution, Prakash ${ }^{14}$ studied the inverted exponential model and Flaih ${ }^{15}$ invented exponentiated inverted Weibull Distribution. Kumaraswamy distribution introduced by Kumaraswamy ${ }^{16}$ is derived from beta distribution after fixing some parameters posses a closed-form cdf which is invertible, moments also exist. This distribution is applicable to many natural phenomena related to which outcomes have lower and upper bounds such as height of individuals, scores obtained on a test, atmospheric temperature, hydrological data such as daily rainfall, daily streamed etc. Alizadeh et al., ${ }^{17}$ introduced Kumaraswamy Marshal-Olkin generalized family of distributions. From this distribution we can derive new generalized model with distinct mathematical and statistical properties. Recently appeared new inverted Kumaraswamy distribution based on transformation $\left(T=1-\frac{1}{X}\right)$ of the Kumaraswamy distribution by Abd AL-Fattah. ${ }^{18}$ This new class of distribution is called inverted Kumaraswamy (IKum) distribution. Also Iqbal ${ }^{19}$ introduced generalized inverted Kumaraswamy distribution by giving another transformation $\left(T=1-\frac{1}{X^{a}}, a>0\right)$ to Kumaraswamy distribution. In this paper we concentrated on an extended form of IKum distribution. A random variable $\mathrm{X}$ is said to have the IKum distribution with parameters with shape parameters $\beta$ and $\gamma$ if it's cdf is given by

$$
F(x, \beta, \gamma)=\left(1-(1+x)^{-\gamma}\right)^{\beta}(x>0, \beta, \gamma>0), x>0, \beta, \gamma>0
$$

and the pdf is as

$$
f(x, \beta, \gamma)=\beta \gamma(1+x)^{-(\gamma+1)}\left(1-(1+x)^{-\gamma}\right)^{\beta-1}, x>0, \beta, \gamma>0 .
$$

From this distribution we can derive a number of sub-models for example, Lomax distribution, Beta type II distribution, log-logistic distribution etc. Also this distribution is flexible enough to model lifetime data. This paper is organized as follows. Section 2 deals with the basic concepts of MOE inverted Kumaraswamy distribution. Its properties including moments, moment generating function, quantiles and mode are presented in Section 3. Section 4 deals with estimation of parameters using maximum likelihood method. Finally, concluding remarks are addressed in Section 5.

\section{MOE iKum distribution}

In this section we consider the cdf of MOEIKum distribution by selecting in (1.1) the generalized inverted Kumaraswamy cdf (1.3) which yields

$$
G(x, \alpha, \beta, \gamma)=\frac{\left(1-(1+x)^{-\gamma}\right)^{\beta}}{\left(\alpha+(1-\alpha)\left(1-(1+x)^{-\gamma}\right)^{\beta}\right)}, x>0, \alpha, \beta, \gamma>0
$$

and, according to (1.2) and (1.4), the related pdf reads 


$$
g(x, \alpha, \beta, \gamma)=\frac{\alpha \beta \gamma(1+x)^{-(\gamma+1)}\left(1-(1+x)^{-\gamma}\right)^{\beta-1}}{\left[\alpha+(1-\alpha)\left(1-(1+x)^{-\gamma}\right)^{\beta}\right]^{2}}, x>0, \alpha, \beta, \gamma>0
$$

The hazard rate function of the MOEIKum distribution is given by following equation

$$
h(x, \alpha, \beta, \gamma)=\frac{\beta \gamma(1+x)^{-(\gamma+1)}\left(1-(1+x)^{-\gamma}\right)^{\beta-1}}{\left[\alpha+(1-\alpha)\left(1-(1+x)^{-\gamma}\right)^{\beta}\right]\left[1-\left(1-(1+x)^{-\gamma}\right)^{\beta}\right]}, x>0, \alpha, \beta, \gamma>0
$$

\section{Shape properties}

Figures 1 illustrate how the tacitly involved new parameter $\alpha$ affect the pdf of generalized inverted Kumaraswamy distribution. The curves tend to flatten as $\alpha$ increases when keeping the other parameters fixed. From this figure, we observe that the MOEIKum pdf can be either a decreasing or a unimodal function. The different shapes of the hazard rate function of the MOEIKum distribution are displayed in Figure 2 for selected parameter values. So, we observe that the MOEIKum hazard rate function can be increasing, decreasing, upside-down bathtub (unimodal) depending on the values of its parameters. A characteristic of the proposed distribution is that its hazard rate function accommodates increasing, decreasing, and unimodal shaped forms, that depend basically on the values of the shape parameters. Moreover, it is quite flexible for modeling survival data. The following theorem describes the shapes of pdf of the MOEIKum distribution.

Theorem 2.1: For $\alpha<1$ the $p d f$ of the MOEIKum distribution is decreasing when $0<\beta<1$.

Proof: The natural logarithm of equation (2.2) is

$I=\log (\alpha \beta \gamma)-(\gamma+1) \log x+(\beta-1) \log \left(1-(1+x)^{-\gamma}\right)-2 \log \left(\alpha+(1-\alpha)\left(1-(1+x)^{-\gamma}\right)^{\beta}\right)$

Now differentiating $I$ with respect to x yields:

$$
I^{\prime}(x)=-\frac{\gamma+1}{1+x}+\frac{(\beta-1) \gamma(1+x)^{-\gamma-1}}{1-(1+x)^{-\gamma}}-2 \frac{\beta \gamma(1-\alpha)\left(1-(1+x)^{-\gamma}\right)^{\beta-1}(1+x)^{-\gamma-1}}{\left[\alpha+(1-\alpha)\left(1-(1+x)^{-\gamma}\right)^{\beta}\right]}
$$

So, from (2.3) it is clear that $I^{\prime}(x)<0$ for all $x>0,0<\beta<1$ and $\alpha<1$. This implies that pdf is decreasing for $0<\beta<1$ and $\alpha<1$.

\section{Related distributions and transformed variables}

Some important sub-models of the MOEIKum distribution are following;

1. Lomax (Pareto Type II) Distribution : when $\alpha=\beta=1$ in equation (2.2) with pdf

$$
g(x, \gamma)=\frac{\gamma}{(1+x)^{\gamma+1}}, x>0, \gamma>0
$$

2. MOE Lomax Distribution: when $\alpha=1$ in equation (2.2) with pdf

$$
g(x, \alpha, \gamma)=\frac{\alpha \gamma(1+x)^{-(\gamma+1)}}{\left[\alpha+(1-\alpha)(1-(1+x))^{-\gamma}\right]^{2}}, x>0, \alpha, \gamma>0
$$

3. Beta Type II Distribution : when $\alpha=\gamma=1$ in equation (2.2) with pdf

$$
g(x, \beta)=\frac{1}{B(\beta, 1)} \frac{x^{\beta-1}}{(1+x)^{\beta+1}}, x>0, \beta>0
$$

4. Log-Logistic (Fisk) Distribution : when $\alpha=\beta=\gamma=1$ in equation (2.2) with pdf

$$
g(x, \beta)=\frac{1}{(1+x)^{2}}, x>0, \beta>0
$$

5. MOE Log-Logistic (Fisk) Distribution : when $\beta=\gamma=1$ in equation (2.2) with pdf

$$
g(x, \alpha, \beta)=\frac{(1+x)^{-2}}{\left[\alpha+(1-\alpha)(1-(1+x))^{-1}\right]^{2}}, x>0, \beta>0
$$

By applying appropriate transformation to variable of MOEIKum distribution, we can derive a number of distributions. These transformation and resulting distributions are given in Table 1. Following theorem mentioned limiting distribution of MOEIKum model.

Theorem 2.2: If $W$ has $M O E$ exponential distribution then the random variable

$$
T=\left[\left(1-e^{w}\right)^{-\frac{1}{\gamma}}-1\right], w, \gamma>0
$$

has MOEIKum distribution.

Proof: Let $W$ follow the MOE exponential distribution with parameter $\beta$, its pdf is given by

$$
f_{w}(w, \alpha, \beta)=\frac{\alpha \beta e^{-\beta w}}{\left\{\alpha+(1-\alpha)\left(1-e^{-\beta w}\right)\right\}^{2}}, w, \alpha, \beta>0
$$

Let $T=\left[\left(1-e^{w}\right)^{-\frac{1}{\gamma}}-1\right], w=-\log \left(1-(1+t)^{-\gamma}\right)$ and $\left|\frac{d w}{d t}\right|=\frac{\gamma(1+t)^{-\gamma-1}}{\left(1-(1+t)^{-\gamma}\right)}$, then the pdf of $T$ is obtained as

Also

$$
f(t)=f_{w}\left[\left(1-e^{w}\right)^{-\frac{1}{\gamma}}-1\right]\left|\frac{d w}{d t}\right|
$$

$$
f(t, \alpha, \beta, \gamma)=\frac{\alpha \beta \gamma(1+t)^{-(\gamma+1)}\left(1-(1+t)^{-\gamma}\right)^{\beta-1}}{\left[\alpha+(1-\alpha)\left(1-(1+t)^{-\gamma}\right)^{\beta}\right]^{2}}, \alpha, \beta, \gamma>0
$$

which is a pdf of MOEIKum $(\alpha, \beta, \gamma)$ distribution.

$\underset{1}{\operatorname{Remark}}$ 1: If $X \sim \operatorname{MOEIKum}(\alpha, \beta, \gamma)$ and $T_{1}=\beta^{-\frac{1}{\gamma}}(1+X)$ on $\left(\beta^{-\frac{1}{\gamma}}, \infty\right)$, then the pdf of $t_{1}$ tends to MOE Inverted Weibull distribution as $\beta \rightarrow \infty$ with pdf

$$
g\left(t_{1}, \alpha, \gamma\right)=\frac{\alpha \gamma t_{1}^{-(\gamma+1)} e^{-t^{-\gamma}}}{\left[\alpha+(1-\alpha) e^{-t^{-\gamma}}\right]^{2}}, t_{1}>0, \alpha, \gamma>0
$$

\section{Compounding}

The following theorem shows that under suitable conditions the MOEIKum distribution can be expressed as compound distribution with exponential distribution as mixing density.

Theorem 2.3: Let $X$ be a continuous random variable with conditional pdf given as

$$
\bar{G}(x \mid \theta)=\exp -\left(\left[1-\left(\left(1-(1+x)^{-\gamma}\right)^{\beta}\right)\right]-1\right) \theta, x, \delta, \beta, \theta>0 .
$$


Let $\Theta$ follows an exponential distribution with pdf given by $m(\theta)=\alpha e^{-\alpha \theta}, \theta, \quad \alpha>0$. Then the compound distribution of $\mathrm{X}$ becomes the MOEIKum $(\alpha, \beta, \gamma)$ distribution.

Proof: For all $x>0, \alpha>0, \beta>0, \gamma>0$, the unconditional survival function of $X$ is given by

$$
\bar{G}(x)=\int_{0}^{\infty} \bar{G}(x \mid \theta) v(\theta) d \theta
$$

$$
\begin{gathered}
=\alpha \int_{0}^{\infty} \exp -\left(\left[1-\left(\left(1-(1+x)^{-\gamma}\right)^{\beta}\right)\right]-1\right) \theta e^{-\alpha \theta} d \theta \\
=\frac{\alpha\left[1-\left(1-(1+x)^{-\gamma}\right)^{\beta}\right]}{\left(1-(1-\alpha)\left(1-\left(1-(1+x)^{-\gamma}\right)^{\beta}\right)\right)}
\end{gathered}
$$

which is the survival function of a random variable with MOEIKum

\begin{tabular}{|c|c|c|}
\hline Transformation & Resulting distribution & pdf \\
\hline$T=[\log (1+x)]^{\frac{1}{\theta}}$ & & \\
\hline special cases: & MOE Exponentiated Weibull & \\
\hline$\theta-1$ & MOE Exponentiated exponential & \\
\hline & MOE Weibull & \\
\hline$\beta=1$ & MOE Burr Type $X$ & $f_{1}(t)=\frac{\alpha \beta \gamma \theta t^{\theta-1} e^{-\gamma t}\left(1-e^{-\gamma t}\right)^{\beta-1}}{}$ \\
\hline$\theta=2$ & MOE Exponential & {$\left[\alpha+(1-\alpha)\left(1-e^{-\gamma t}\right)^{\beta}\right]^{2}$} \\
\hline$\beta=\theta=1$ & Exponentiated exponential & $0<t<\infty$ \\
\hline$\alpha=1, \theta=1$ & Weibull & \\
\hline$\alpha=\beta=1$ & $\begin{array}{l}\text { Burr Type X } \\
\text { Exponential }\end{array}$ & $\alpha, \beta, \gamma>0$ \\
\hline$\alpha=1, \theta=2$ & & \\
\hline$\alpha=\beta=\theta=1$ & & \\
\hline$\underline{1}$ & & \\
\hline$T=r^{\bar{\gamma}}(1+X)^{-1}+s$ & MOE Generalized uniform & \\
\hline special cases: & Generalized uniform & $f_{2}(t)=\alpha \beta \gamma r(t-s)^{\gamma-1}\left[1-r(t-s)^{\gamma}\right]^{\beta-1}$ \\
\hline$\alpha=1$ & Beta Type I & $\begin{array}{l}\alpha+(1-\alpha)\left[1-r(t-s)^{\gamma}\right]^{\beta} \\
-\frac{1}{2}\end{array}$ \\
\hline$\alpha=\gamma=s=1$ & Inverted generalized Pareto Type I & $s<t<s+r^{-\bar{\alpha}}$ \\
\hline $\begin{array}{l}\alpha=1, s=0 \\
\alpha=\gamma=\beta=r=1\end{array}$ & Uniform $(0,1)$ & $\alpha, \beta, \gamma, r, s>0$ \\
\hline $\begin{array}{l}T=\log (1+T)^{\theta}+b \\
\text { special cases : } \\
\alpha=\beta=1\end{array}$ & $\begin{array}{l}\text { Left truncated exponentiated exponential } \\
\qquad \text { Left truncated exponential }\end{array}$ & $f_{3}(t)=\frac{\gamma \beta}{\theta} e^{-\gamma\left[\frac{t-b}{\theta}\right]}\left(1-e^{-\alpha \frac{t-b}{\theta}}\right)^{\beta-1}$ \\
\hline$\alpha=\beta=1, b=0$ & exponential & $b<t<\infty$ \\
\hline$T=s X^{\frac{1}{c}}$ & $\begin{array}{l}\text { MOE Exponentiated Burr Type XII } \\
\text { MOE Burr Type XII }\end{array}$ & $l(t)=\frac{\gamma \beta c}{s^{c}} t^{c+1}\left[1+\left(\frac{t}{s}\right)^{c}\right]^{-(\gamma+1)}$ \\
\hline specialcases: & MOE Generalized Lomax & $\left(1-\left[1+(\stackrel{t}{-})^{c}\right](-\gamma)\right)^{\beta-1}$ \\
\hline$\alpha=\beta=1$ & MOE Exponentiated Burr Type XII & \\
\hline$\alpha=\beta=c=1$ & Burr Type XII & $f_{4}(t)=\frac{l(t)}{r}$ \\
\hline $\begin{array}{l}\alpha=\beta=c=s=1 \\
\alpha=\beta=c=1, s=\alpha\end{array}$ & $\begin{array}{l}\text { Generalized Lomax } \\
\text { Beta Type II } \\
\text { F-distribution }\end{array}$ & $\begin{array}{l}{\left[\alpha+(1-\alpha)\left(1-\left[1+\left(\frac{t}{s}\right)^{c}\right](-\gamma)\right)^{\beta}\right.} \\
t>0, \alpha, \beta, \gamma, s, c>0\end{array}$ \\
\hline
\end{tabular}
distribution.

Table I Transformations Applied to MOEIKum and the Resulting Distributions 
Table Continued

\section{Transformation}

$T=\left[\frac{X}{\lambda}\right]^{-\frac{1}{s}}, \alpha=1$

specialcases :

$$
\begin{gathered}
\beta=\lambda=1 \\
\gamma=1
\end{gathered}
$$

$T=\left(\frac{\log (1+X)}{\theta}\right)\left(-\frac{1}{b}\right), \alpha=1$

specialcases :

$b=1$

$b=\beta=1$
Resulting distribution

pdf

Kumaraswamy-Dagum

Dagum

Kumaraswamy-Burr Type III

Burr Type III

Kumaraswamy-Fisk

Kumaraswamy-inverse Weibull

Kumaraswamy-inverse exponential

$t>0$
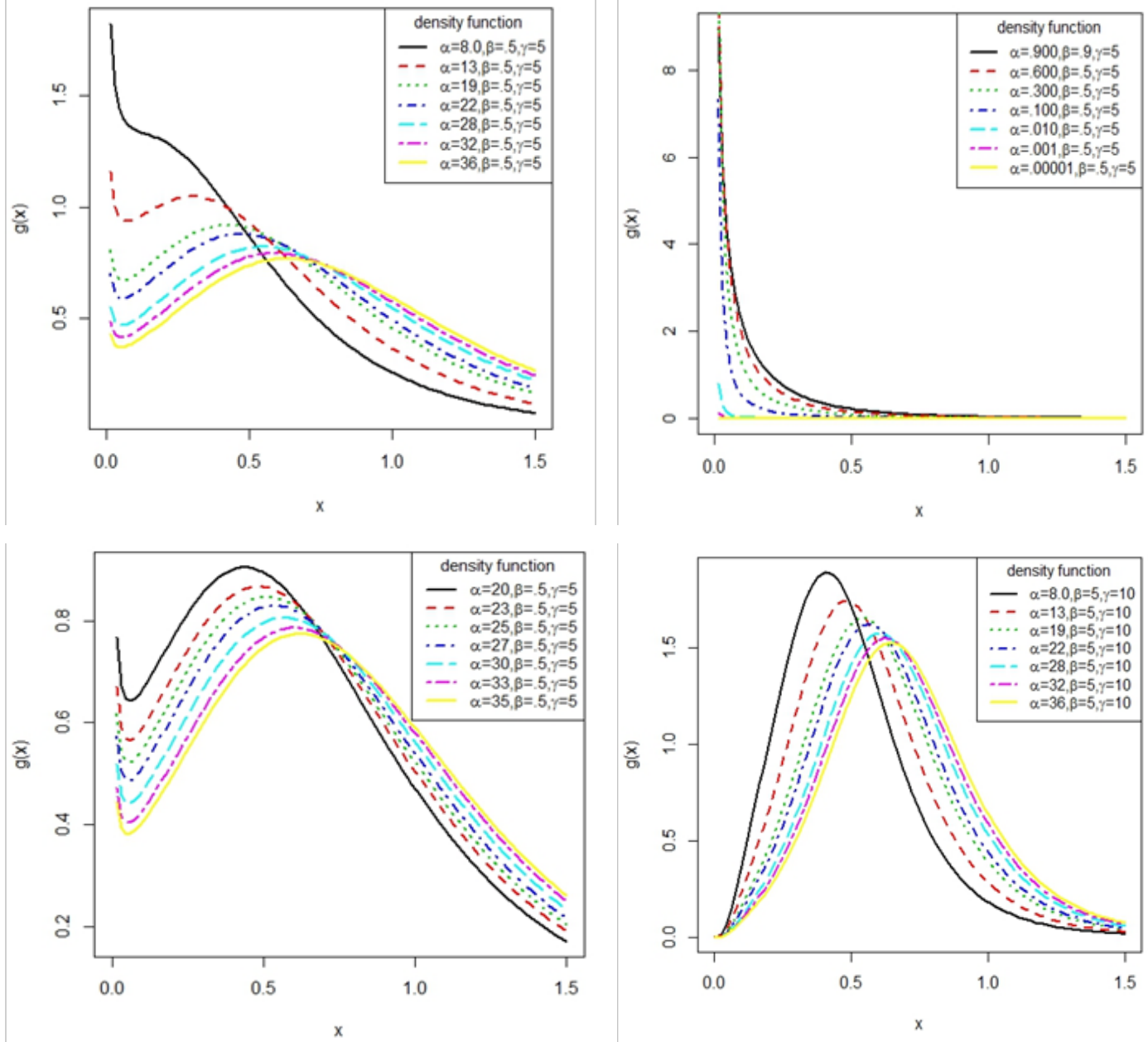

Figure I Graphs of pdf of the MOEIKum distribution for different values of $\alpha, \beta$ and $\gamma$. '

${ }^{1}$ The MOEIKum pdf can be either a decreasing(when $\alpha<1$ ) or a unimodal function. 

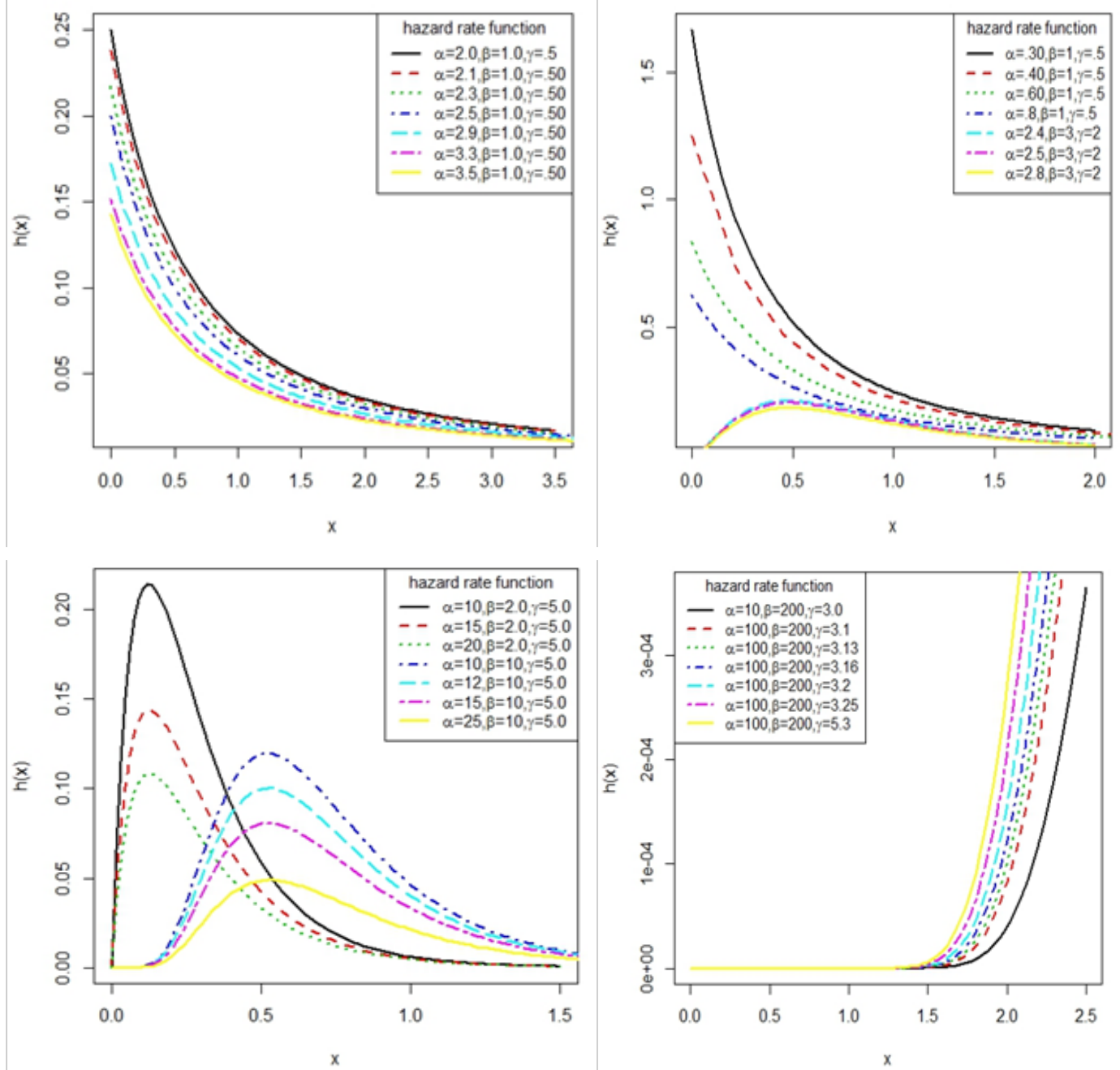

Figure 2 Graphs of pdf of the MOEIKum distribution for different values of $\alpha, \beta$ and $\gamma .{ }^{22}$

${ }^{2}$ The MOEIKum hazard rate function can be increasing, decreasing, upside-down bathtub (unimodal) depending on the values of parameters

\section{Properties of MOEIKum distribution}

\section{Moments and moment generating function}

We now give simple expansions for the pdf of the MOEIKum distribution. We have generalized binomial expansion,

$$
(1-z)^{-r}=\sum_{i=0}^{\infty}\left(\begin{array}{l}
r+i-1 \\
i
\end{array}\right) z^{i},|z|<0
$$

Using the series expansion (3.1) and the representation for the MOEIKum pdf (2.2), we obtain

$$
\begin{gathered}
g(x)=\alpha \sum_{i}^{\infty} \sum_{j}^{\infty}(-1)^{j} i(1-\alpha)^{i}\left(\begin{array}{c}
i \beta+j-1 \\
j
\end{array}\right) \beta \gamma(1+x)^{-(\gamma+1)}\left(1-(1+x)^{-\gamma}\right)^{\beta j+\beta-1} \\
=\sum_{i}^{\infty} \sum_{j}^{\infty}(-1)^{j} i(1-\alpha)^{i}\left(\begin{array}{c}
i \beta+j-1 \\
j
\end{array}\right) \operatorname{Ikum}(\beta, \gamma) \\
=\alpha \beta \gamma \sum_{i}^{\infty} \sum_{j}^{\infty} \sum_{k}^{\infty}(-1)^{j+k} i(1-\alpha)^{i} \\
\left(\begin{array}{l}
i \beta+j-1 \\
j
\end{array}\right)\left(\begin{array}{l}
k+\beta j+\beta-2 \\
k
\end{array}\right)(1+x)^{-k \gamma-\gamma-1}
\end{gathered}
$$

From equation (3.2), it is clear that the pdf of MOEIKum can be expressed as an infinite linear combination of IKum pdfs.

$$
E(x)^{r}=\int_{0}^{\infty} X^{r} g(x, \alpha, \beta, \gamma) d x .
$$

Substituting (3.3) into the equation (3.4) yields

$$
\begin{gathered}
E(x)^{r}=\alpha \beta \gamma \sum_{i}^{\infty} \sum_{j}^{\infty} \sum_{k}^{\infty}(-1){ }^{j+k} i(1-\alpha)^{i}\left(\begin{array}{c}
j+i \beta-1 \\
j
\end{array}\right)\left(\begin{array}{l}
k+\beta j+\beta-2 \\
k
\end{array}\right) \int_{0}^{\infty} x^{r}(1+x)^{-k \gamma-\gamma-1} d x \\
=\sum_{i}^{\infty} \sum_{j}^{\infty} \sum_{k}^{\infty} M_{i, j, k} B(r+1, k \gamma+\gamma-r)
\end{gathered}
$$

where $\mathrm{B}$ is beta function and

$$
M_{i, j, k}=\alpha \beta \gamma i(-1)^{j+k}(1-\alpha)^{i}\left(\begin{array}{c}
j+i \beta-1 \\
j
\end{array}\right)\left(\begin{array}{l}
k+\beta j+\beta-2 \\
k
\end{array}\right) \text {. }
$$

Moment generating function given by following formula

$$
\mathrm{M}_{X}(t)=E\left(e^{t X}\right)=\sum_{r=0}^{\infty} \frac{t^{r}}{r !} E\left(X^{r}\right)
$$

where $E\left(X^{r}\right)$ follows from (3.5). The Mean,Variance, Skewness and Kurtosis can be obtained from the equation (3.7). 


\section{Quantiles and mode}

The $p^{\text {th }}$ quantile function of the distribution, the inverse of the distribution function $F\left(x_{p}\right)=p$, is given by

$$
x=\left\{1+\left[\frac{p \alpha}{1-p-p \alpha}\right]^{\frac{1}{\beta}}\right\}^{-\frac{1}{\gamma}}-1 .
$$

$p=1 / 2$ (the second quantile) yields the median given by

$$
\text { Median }=\left\{1+\left[\frac{\alpha}{1-\alpha}\right]^{\frac{1}{\beta}}\right\}^{-\frac{1}{\gamma}}-1 .
$$

From (2.3), mode of this distribution can be found by solving $\frac{\partial I}{\partial x}=0 \quad\left(\frac{\partial^{2} I}{\partial x^{2}}<0\right)$.

\section{Estimation of parameters}

In this section we consider maximum likelihood estimation for a given sample of size $x_{1}, x_{2}, \ldots, x_{1}$ from $\operatorname{MOEIKum}(\alpha, \beta, \gamma)$, then the log likelihood function is given by

$$
\begin{gathered}
\log L(\alpha, \beta, \gamma)=n \log (\alpha \beta \gamma)-(\gamma+1) \sum_{i=1}^{n} \log \left(1+x_{i}\right)+(\beta-1) \sum_{i=1}^{n} \log \left(1-\left(1+x_{i}\right)^{-\gamma}\right) \\
-2 \sum_{i=1}^{n} \log \left(1-\bar{\alpha}\left[1-\left[1-\left(1+x_{i}^{-\gamma}\right)\right]^{\beta}\right]\right)
\end{gathered}
$$

The partial derivative of the log likelihood functions with respect to the parameters are

$$
\begin{gathered}
\frac{\partial \log L}{\partial \alpha}=\frac{n}{\alpha}-2 \sum_{i=1}^{n} \frac{1-\left[1-\left(1+x_{i}^{-\gamma}\right)\right]^{\beta}}{\left.-\alpha 1-\left[1-\left(1+x_{i}^{-\gamma}\right)\right]^{\beta}\right]} \\
\frac{\partial \log L}{\partial \beta}=\frac{n}{\beta}+\sum_{i=1}^{n} \log \left(1-\left(x_{i}+1\right)^{-\gamma}\right)-2 \bar{\alpha} \sum_{i=1}^{n} \frac{\left[1-\left(1+x_{i}^{-\gamma}\right)\right]^{\beta} \log \left(1-\left[1+x_{i}^{-\gamma}\right]\right)}{1-\alpha\left[1-\left[1-\left(1+x_{i}^{-\gamma}\right)\right]^{\beta}\right]} \\
\frac{\partial \log L}{\partial \gamma}=\frac{n}{\gamma}+\sum_{i=1}^{n} \log \left(x_{i}\right)+(\beta-1) \sum_{i=1}^{n} \frac{\left[1-\left(1+x_{i}^{-\gamma}\right)\right] \log \left(1-\left(1+x_{i}^{-\gamma}\right)\right)}{\left[1-\left(1+x_{i}^{-\gamma}\right)\right]} \\
-2 \sum_{i=1}^{n} \frac{\left[1-\left(1+x_{i}^{-\gamma}\right)\right] \log \left(1-\left(1+x_{i}^{-\gamma}\right)\right)}{1-\alpha\left[1-\left[1-\left(1+x_{i}^{-\gamma}\right)\right]^{\beta}\right]}
\end{gathered}
$$

The maximum likelihood estimates can be numerically obtained by solving the equations $\frac{\partial \log L}{\partial \alpha}=0, \frac{\partial \log L}{\partial \beta}=0, \frac{\partial \log L}{\partial \gamma}=0$ For this purpose, we can use functions like nlm, fitdist or optimize from the programming language $R$.

\section{Conclusions}

In this paper, MOEIKum distribution based on the MarshallOlkin method of generalization has been proposed and its properties are studied. This new model could have increasing, decreasing and unimodal pdf and it has an inverted bathtub hazard rate. The behavior of the hazard rate function shows that the proposed model is applicable in situations where the Kumaraswamy distribution and the IKum distribution are used and can as well serve as better alternative to both of them. The proposed model enfolds a number of special sub models and also get some existing distribution by adding appropriate transformation to this model. This distribution can be expressed as compound distribution with exponential distribution as mixing density and also the MOE exponential distribution and the MOE Inverted Weibull distribution can be obtained as a limiting distribution of this model.

\section{Acknowledgements}

The second author is grateful to the Department of Science and Technology (DST), Govt. of India for the financial support under the INSPIRE Fellowship.

\section{Conflict of interest}

None

\section{References}

1. Gupta RC, Gupta PL, Gupta RD. Modeling Failure Time Data by Lehman Alternatives. Communications in Statistics- Theory and Methods. 1998;27:887-904.

2. Cordeiro GM, Castro M. A New Family of Generalized Distributions. Journal of Statistical Computation and Simulation. 2011;81:883-898.

3. Pescim RR, Cordeiro GM, Demétrio CGB, et al. The new class of Kummer beta generalized distributions. SORT. 2012;36(2):153-180.

4. Nadarajah S, Cancho VG, Ortega EMM. The geometric exponential Poisson distribution. Statistical Methods and Applications. 2013;22(3):355-380.

5. Alzaghal A, Famoye F, Lee C. Exponentiated T-X family of distributions with some applications. International Journal of Probability Statistics. 2013;2(3):31-49.

6. Bourguignion M, Silva RB, Cordeiro GM. The Weibull Generalized family of probability distributions. Journal of Data Science. 2014;12:53-68

7. Nadarajah S, Teimouri M, Shih SH. Modified beta distributions. Sankhya. 2014;76:19-48.

8. Risti MM, Nadarajah S. A new lifetime distribution. Journal of Statistical Computations and Simulations. 2014;84(1):135-150.

9. Marshall AW, Olkin I. A new method for adding a parameter to a family of distributions with application to the exponential and Weibull families. Biometrica. 1997;84:641-652.

10. Calabria R, Pulcini G. On the maximum likelihood and least squares estimation in the inverse Weibull distribution. Journal of Statistica Applicate. 1990;2:53-66.

11. AL Dayian GR. Burr Type III distribution: Properties and estimation. The Egyptian Statistical Journal. 1999;43:102-116.

12. Abd EL Kader RI, AL Dayian, et al. Inverted Pareto Type I distribution: properties and estimation. Journal of Faculty of Commerce AL Azhar University Girls'Branch. 2003;21:19-40.

13. AL Dayian GR. Inverted Pareto Type II distribution: Properties and estimation. Journal of Faculty of Commerce AL-Azhar University Girls Branch. 2004;22:1-18.

14. Prakash G. Inverted exponential distribution under a Bayesian view point Journal of Modern Applied Statistical Methods. 2012;11(1):190-202.

15. Flaih A, Elsalloukh H, Mendi E, et al. The Exponentiated Inverted Weibull Distribution. Applied Mathematics and Information Sciences. 2012;6:167-171.

16. Kumaraswamy P. A generalized probability density function for doublebounded random processes. Journal of Hydrology. 1980;46(1-2):79-88. 
17. Alizadeh M, Tahir MH, Cordeiro GM, et al. The Kumaraswamy Marshal-Olkin family of distributions. Journal of the Egyptian Mathematical Society. 2015;23(3):546-557.

18. Abd AL Fattah AM, EL Helbawy AA, AL Dayian GR. Iverted Kumaraswamy Distribution: Properties and Application. Pakistan Journal of Statistics. 2017;33(1):37-61.
19. Iqbal Z, Tahir MM, Riaz N, et al. Generalized Inverted Kumaraswamy Distribution: Properties and Application. Open Journal of Statistics. 2017;7:645-662. 